\title{
baRcodeR with PyTrackDat: Open-source labelling and tracking of biological samples for repeatable science
}

\author{
PREPRINT
}

baRcodeR is now available for R: https://cran.r-project.org/package=baRcodeR

It can be installed directly in the R environment using the command: install.packages( 'baRcodeR' )

PyTrackDat is still in development, as of October 29, 2018, but available on GitHub https://github.com/ColauttiLab/PyTrackDat

Yihan Wu, David R. Lougheed, Stephen C. Lougheed, Kristy Moniz, Virginia K. Walker, and Robert I. Colautti*

\section{Biology Department}

Queen's University

116 Barrie St.

Kingston, ON K7L 3N6

*Corresponding Author Email: robert.colautti@queensu.ca

Keywords: Biological samples; asset tracking; inventory management; database; barcodes 
bioRxiv preprint doi: https://doi org/10.1101/457051; this version posted October 30, 2018. The copyright holder for this preprint (which was not certified by peer review) is the author/funder, who has granted bioRxiv a license to display the preprint in perpetuity. It is made available under aCC-BY-NC-ND 4.0 International license.

baRcodeR with PyTrackDat

\section{Abstract}

Repeatable experiments with accurate data collection and reproducible analyses are fundamental to the scientific method but may be difficult to achieve in practice. Several flexible, open-source tools developed for the $\mathrm{R}$ and Python coding environments aid the reproducibility of data wrangling and analysis in scientific research. In contrast, analogous tools are generally lacking for earlier stages, such as systematic labelling and processing of field samples with hierarchical structure (e.g. time points of individuals from multiple lines or populations) or curating heterogenous data collected by different researchers over several years. Such tools are critical for modern research given trends toward globally distributed collaborators using higher-throughput technologies. As a step toward improving repeatability of methods for the collection of biological samples, and curation of biological data, we introduce the R package baRcodeR and the PyTrackDat pipeline in Python. The baRcodeR package provides tools for generating biologically informative, hierarchical labels with digitally encoded 2D barcodes that can be printed and scanned using low-cost commercial hardware. The PyTrackDat pipeline integrates with baRcodeR output to build a web interface for sample management and tracking along with data collection and curation. We briefly describe the application of principles from baRcodeR and PyTrackDat in three large research projects, which demonstrate their value to (i) help document sampling methods, (ii) facilitate collaboration and (iii) reduce opportunities for human errors and omissions that could otherwise propagate through downstream data analysis to compromise biological inference.

\section{Introduction}

The increasing use of high-throughput methods for data collection and popularity of large collaborative research projects in biology poses challenges for researchers tracking samples and their associated data across field and laboratory experiments. New standards and tools for documenting data analysis have developed alongside rapid advancements in computation and global communication, aimed at improving repeatability, reproducibility, accuracy and accountability of published scientific research. These methods are likely to become more prevalent as major funding bodies move toward principles and policies stressing well-developed data management plans (DMPs), as demonstrated in the Draft TriAgency Research Data Management Policy (Government of Canada), Article 29 of the ERC Multi-Model Grant Agreement (European Commission), and the NSF open data policy (National Science Foundation).

A push towards more transparent, reproducible data workflows for scraping, merging, cleaning, editing, error checking, pre-processing and curating data for subsequent visualization and statistical analysis (i.e. data wrangling) has inspired the rapid development of new tools. For example, $R$ markdown and $R$ notebooks with R Studio (RStudio Team, 2016) are now common tools to facilitate careful documentation of data visualization and statistical analysis in $R$ ( $R$ Core Team, 2018). Jupyter Notebooks and Jupyter Lab (Kluyver et al., 2016) serve similar functions for reproducible analysis in Python (Oliphant, 2007). Such tools allow for fully reproducible data wrangling and analysis from a starting dataset, which was difficult or impossible with previous generations of proprietary spreadsheet databases and point-and-click statistical packages. In contrast, tools to improve transparency, accuracy, repeatability, and reproducibility at the earlier stages of sampling, data collection and curation have received relatively little attention, even though modern downstream management packages assume accurate sample tracking and recording of raw data and sampling details.

In many of the biological sciences, samples are collected under arduous field conditions that pose challenges for labelling, organizing, measuring and analyzing samples. A common approach is to quickly collect and preserve samples for later analysis. This often requires transport of samples to different locations, to different storage media or vessels, and/or subsampling for multiple analyses. An individual 
bioRxiv preprint doi: https://doi.org/10.1101/457051; this version posted October 30, 2018. The copyright holder for this preprint (which was not certified by peer review) is the author/funder, who has granted bioRxiv a license to display the preprint in perpetuity. It is made available under aCC-BY-NC-ND 4.0 International license.

baRcodeR with PyTrackDat

biological sample is often tied to a variety of heterogenous data such as locations, images, morphological measurements, behavioral assays, chemical analysis, as well as details about the sampling method itself, such as the identities of individuals collecting, processing, and measuring the samples. Heterogeneous data may be collected by different individuals or labs and stored in a variety of formats but are inherently linked by details about their collection such as sampling method, date and location, collector identity, and other metadata. Misinterpreted labels, cut-and-paste errors, typographical and scribing errors, and other problems that arise when humans associate samples with their downstream measurements and metadata can potentially go undetected and propagate through statistical analysis to compromise biological inference.

Asset tracking using digital barcodes can significantly reduce opportunities for human error by automating and/or acting as additional error checks on human tasks that are error prone (Copp et al., 2014). Indeed, this 'industrial approach' has been successfully adopted to improve cataloguing of natural history collections that may contain thousands to millions of specimens (Blagoderov et al., 2012). Perhaps the strongest evidence for the value of digital barcodes is their ubiquitous use in inventory tracking and sales in virtually every type of commerce on earth, ranging from perishable foods with short turnaround times to inventories in long-term storage facilities. In addition to reducing human error, digital barcoding technology can simplify data collection when combined with data collection software and a dedicated barcode scanner or image recognition applications for smartphone, tablet, or personal computers.

Numerous options are available for asset tracking using barcodes, many with integrated databases (e.g. ManageEngine, Pulseway, Asset Panda, GoCodes, OpenLab Framework). However, most of these solutions are proprietary and can be expensive (but see List et al., 2014). These programs prioritize easeof-use, employing a graphical-user interface (GUI) that are relatively easy to learn but can limit options for customization, automation and integration with downstream data wrangling and analysis. Whereas numerous statistical packages exist that improve transparent and reproducible analysis (e.g. in $\mathrm{R}$ and Python) compared to point-and-click statistical software, we know of no analogous options for sample labelling and data collection. Integration of such options within the $\mathrm{R}$ and Python coding environments would facilitate documentation of data management from initial collection through to final analysis for more repeatable and reproducible research.

Here we introduce an integrated programming solution using the baRcodeR package in $\mathrm{R}$ with the PyTrackDat pipeline for labelling and tracking biological samples as well as collecting and curating their associated data. Our goal was to establish a workflow that is (i) open-source, (ii) based in R and Python, (iii) flexible, (iv) inexpensive to implement at scale, and (v) developed specifically for integration within a transparent and robust data management plan for repeatable and reproducible science. To demonstrate the utility of the software and the implementation of the workflow, we include three current case-use studies at different stages of development.

\section{Methods \& Results}

Initial surveys of commercially available digital barcode systems suggested that they were expensive or impractical for our needs. For example, although grocery store hand-held scanners are cheap and robust enough for fieldwork, commercial inventory software could not be easily adapted to suit data collection in the field. Our solution was to write the R package baRcodeR (Wu and Colautti, 2018a) and to extend its functionality with PyTrackDat a Python data pipeline for sample tracking and data collect we call (https://github.com/ColauttiLab/PyTrackDat). 
baRcodeR with PyTrackDat

\section{1. baRcodeR}

The baRcodeR package for $\mathrm{R}$ has a detailed tutorial-style vignette (Wu and Colautti, 2018b) and a 2-page

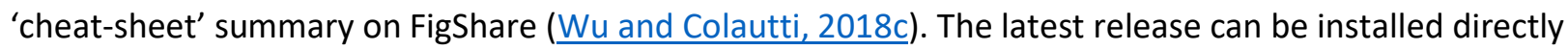
in $\mathrm{R}$ using the command:

\section{> install.packages('baRcodeR')}

A pre-release of the latest features can be installed from GitHub (https://github.com/yihanwu/baRcodeR), either by manually downloading and installing the binaries or using the devtools package:

$>$ devtoools: : install_github('baRcodeR')

Briefly, baRcodeR was developed as an open-source tool for creating digital 2D barcodes in R, making several design choices based on our experiences collecting and managing biological samples. Three main commands are available: (i) uniqID_maker() or (ii) uniqID_hier_maker() are functions to generate unique ID codes, followed by (iii) create_PDF() to output a portable document file (PDF) containing both text labels and 2D barcode images laid out in a customizable format suitable for consumer-grade printers. These functions can be run directly from the command line or via the baRcodeR graphical user interface (GUI) available from the 'Addins' menu in R Studio. The command-line version also includes a more userfriendly interactive mode implemented with the parameter user $=T$.

\section{Unique identifier codes}

To generate standardized and biologically informative ID codes for biological samples, uniqID_maker() or uniqID_hier_maker() are used for sequential or hierarchical text labels, respectively. The uniqID_maker() command is particularly useful to quickly produce sequential labels with the same user-defined prefix string (e.g. Ex-1,Ex-2,Ex-3,...). Additionally, it is possible to pass any sequence of numbers into uniqID_maker(). For example, the user can specify alternating numbers (e.g. Ex-001, Ex-003, Ex$005, \ldots)$ :

$$
>\text { uniqID_maker(string }={ }^{\prime} \text { Ex' }, \text { level }=\operatorname{seq}(1,6,2) \text { ) }
$$

or other custom sequences (e.g. Ex-010,Ex-014,Ex-018,Ex-020,Ex-040):

$$
>\text { uniqID_maker(string }=' \text { Ex', } \operatorname{level}=c(\operatorname{seq}(10,20,4), 20,40))
$$

or randomly ordered sequences:

$$
>\text { uniqID_maker }(\text { string }=\text { 'Ex', level }=\operatorname{sample}(1: 10, \text { replace }=F) \text { ) }
$$

The uniqID_hier_maker() command expands on uniqID_maker(), allowing for the creation of hierarchical labels with different user-defined strings and numeric sequences for each level of the hierarchy. This is useful, for example, to generate labels for replicated populations, genetic lines nested within populations, and repeated sampling or subsampling of individuals at different time points, with randomly assigned treatment categories. For example, one can quickly define labels for ten replicate individuals, each from one of three lines, with subsamples of two different tissue types with the command:

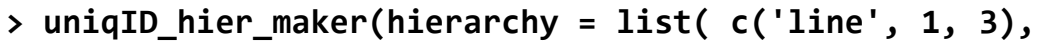

$$
\begin{aligned}
& \left.\left.c\left(\text { 'ind' }^{\prime} 1,10\right), \mathrm{c}\left(\text { 'sbsmpl' }^{\prime} 1,2\right)\right)\right)
\end{aligned}
$$


baRcodeR with PyTrackDat

The examples of uniqID_maker() and uniqID_hier_maker() use non-interactive mode by default, but can also be run interactively in the command line with the user $=T$ parameter. In this mode, the user is prompted for input:

\begin{tabular}{|c|c|c|c|}
\hline \multirow{2}{*}{\multicolumn{4}{|c|}{$\begin{array}{l}\text { Please enter string for level: Ex } \\
\text { Enter the starting number for level }\end{array}$}} \\
\hline & & & \\
\hline \multirow{2}{*}{\multicolumn{4}{|c|}{$\begin{array}{l}\text { Enter the ending number for level: } \\
\text { Number of digits to print for level }\end{array}$}} \\
\hline & & & \\
\hline & 1abe1 & ind_string & ind_number \\
\hline 1 & Ex001 & EX & 1 \\
\hline 2 & Ex002 & Ex & 2 \\
\hline 3 & Ex003 & EX & 3 \\
\hline 4 & Ex004 & EX & 4 \\
\hline 5 & Ex005 & EX & 5 \\
\hline 6 & Ex006 & Ex & 6 \\
\hline 7 & Ex007 & Ex & 7 \\
\hline 8 & Ex008 & EX & 8 \\
\hline 9 & Ex009 & EX & 9 \\
\hline 10 & Ex010 & Ex & 10 \\
\hline
\end{tabular}

Regardless of mode (i.e. interactive or non-interactive), the output of uniqID_maker() and uniqID_hier_maker() is a data.frame object containing (i) a vector of unique IDs and (ii) additional columns corresponding to each level of the sampling hierarchy. Like any data.frame object in $\mathrm{R}$, the output can be renamed with names() function. The output object can also be saved to a standard text file using the write.table() or write.csv() functions. These text files can then be imported into a spreadsheet or database software, or integrated with PyTrackDat (see section 3, below) as part of a documented research workflow.

\section{Printable barcodes}

A data.frame containing a vector of ID labels is required input for the create_PDF() function. The input data.frame may be created by one of the functions above or supplied by the user, for example by entering ID codes manually into a spreadsheet, exporting to CSV, and then importing the CSV file into R using the read.csv() command.

The output of create_PDF() is a printable PDF file containing human-readable (i.e. plain text) IDs and 2-D digital barcode images. These labels can be printed on consumer-grade printers and scanned with standard barcode scanners or image recognition software for cellphones or other devices(Fig. 1). The specific print layout can be customized using spacing parameters in create_PDF(), but default parameters create a PDF output file that will fit S-19297 labels (ULINE.ca or ULINE.com). This layout was chosen because the S-19297 labels are weatherproof vinyl labels ( 80 per page) that can be printed on a standard laser printer. Our own tests demonstrate that printed labels do not fade or degrade even after two years of storage at $-80^{\circ} \mathrm{C}$ or three years of exposure to sunlight in outdoor field experiments. Standard laser printer toner is similarly robust to ultraviolet light and freezing to ultralow temperatures. The label glue is less robust but transparent packing tape can be used to better secure the labels without affecting their function.

The create_PDF() function can be run interactively with the user $=T$ parameter or in default noninteractive mode with user-defined parameters for custom label layouts and other parameters like file 
name and font size. One parameter of particular note is the error correction level $(L, M, Q$ or $H)$, which represent a trade-off between barcode size and redundancy, ranging from larger, high-redundancy $(\mathrm{H})$ to smaller low-redundancy (L) 2-D barcodes. H-codes can still be recognized by scanners after losing up to $30 \%$ of their surface while L-codes labels are less robust but can be printed at a small size. Custom page layout, label layout and printing options can be specified in by passing in arguments through create_PDF() to custom_create_PDF(). See the baRcodeR vignette for details (Wu \& Colautti 2018b).
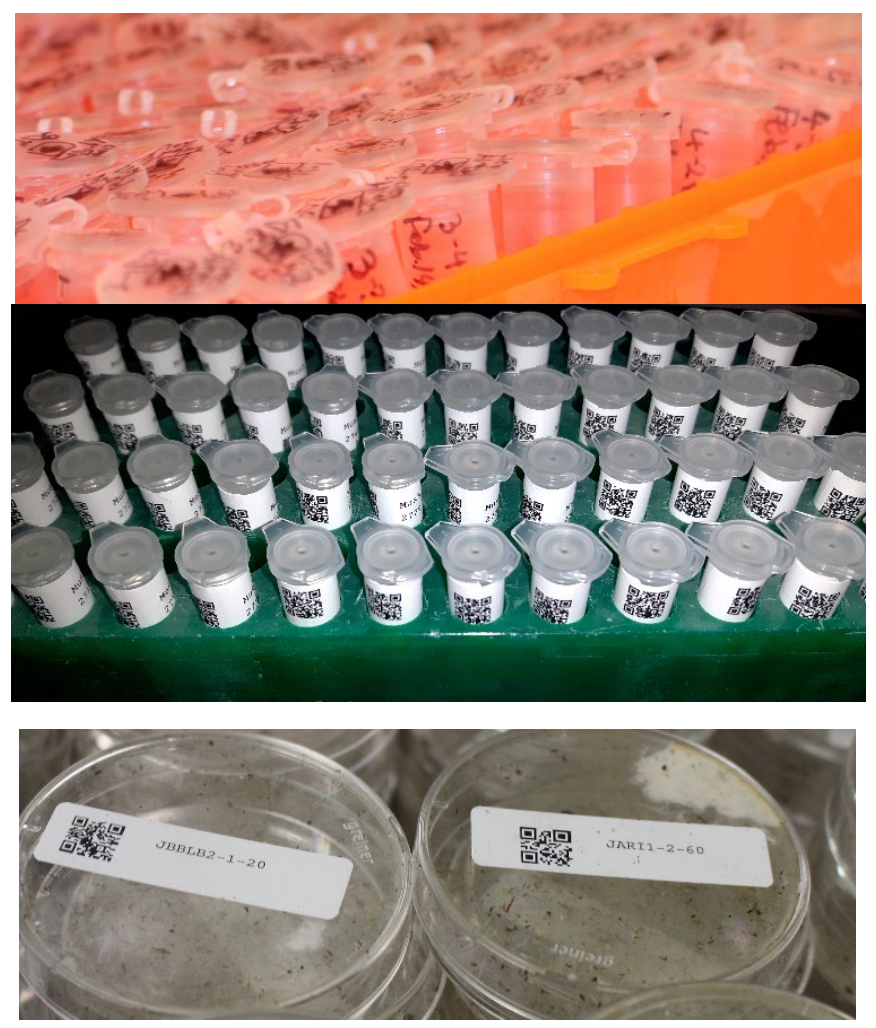

Figure 1. Examples of conventional, hand-labelled tubes (top panel), compared with tubes (mid panel) and petri dishes (lower panel) labelled with digital barcodes and human-readable text labels created using the baRcodeR package.

\section{Graphical user interface}

In addition to the text commands reviewed above, a graphical-user interface (GUI) is available after installing and loading the baRcodeR library (RStudio, 2015). Details of the GUI are provided as a quickstart guide in the README file on the CRAN repository (Wu \& Colautti 2018a; direct link: https://cran.rproject.org/package=baRcodeR/readme/README.html). The GUI add-in is a shiny application (Chang et al., 2018) that can be run directly from the baRcodeR GUI under the 'Addins' menu on the RStudio toolbar. Three tabs in the GUI window correspond to three main functions: 'Simple Label Creation' calls uniqID_maker(), 'Hierarchical Label Creation' calls uniqID_hier_maker(), and 'Barcode Generation' calls create_PDF(). Labels generated from the first two tabs are previewed before final generation and will be automatically saved in the $\mathrm{R}$ working directory upon creation. All advanced parameters for PDF layout are similarly available under the "Barcode Generation" tab. Users can also preview the layout of individual labels before creation. Code snippets are shown for user-specified parameters and can be exported and archived as part of a repeatable experimental design and reproducible analysis. 
baRcodeR with PyTrackDat

In addition to using create_PDF() to generate printable barcodes, users can load saved labels into other barcode printers, such as those that print directly on sample tubes, plant tags, and other material. For example, the TubeWriter 360 (https://tubewriter.com/) and TubeMarker 2 (https://www.4ti.co.uk/) can generate and print barcodes from standard text files, allowing users to create ID codes in uniqID_maker() or uniqID_hier_maker() and saving the output to a text file using the write.table() or write.csv() functions in base $\mathrm{R}$.

Once a data.frame or CSV file is created, additional headings can be added for data entry. Manual data entry is inherently susceptible to human error, but enforcing and restricting entry formats can help to ensure repeatability of scientific inference. A variety of software is available to help reduce errors in data entry, ranging from fully-featured propriety software suites with optical recognition of handwritten data such as Viking Data Entry (http://vikingsoft.com), to open-access programs such as Epidata (Lauritsen and Bruus, 2003) and OpenRefine (http://www.openrefine.org). Microsoft Excel, Libreoffice Calc, and Google Sheets also have data form creators for restricting data entry types. Within RStudio, interactive data entry can be performed using the editData add-in (Moon 2017), which can be appended to the biological sample data.frame created from baRcodeR. Alternatively, users may wish to implement the semi-automated PyTrackDat pipeline to build an online relational database as described in the next two sections.

\section{Data Standards}

Perhaps the simplest implementation of a robust data collection workflow is a 2-dimensional spreadsheet (i.e. data table) containing $n$ rows by $c$ columns. This format has several important properties and considerations, as shown in the example in Figure 2, outlined in Table 1, and discussed below (see also Borer et al., 2009; British Ecological Society, 2014). In this format, each column is a characteristic or measurement and each row is (usually) an individual, with each individual cell containing a single value. To facilitate reproducible analysis, spreadsheet data should not contain empty spaces or formatted text (e.g. colour, bold, italic, underline). Rather, individual values can be repeated, and missing values can be encoded as needed (e.g. NA or NULL). Variable names should be chosen carefully to be as short as possible but still informative. A good strategy for more complicated names is to remove vowels or to use short forms separated by capital letters, underscore or period. For example, a column containing height measurements on day 5 of an experiment might be named HtD5 or Hght.d5. Quotation marks, spaces, commas, semicolons and other common separator characters should be avoided as these are often used by programmers and software to parse data into separate rows or columns. Where necessary, for example in a 'notes' column, these characters can be used in a phrase denoted by quotation marks (e.g. "This is a note; it can be included, if needed, in a 'notes' cell"). 
baRcodeR with PyTrackDat

\section{(A)}

\begin{tabular}{|r|r|l|}
\hline \multicolumn{3}{|c|}{ Date: July 1, 1984} \\
\hline \multicolumn{3}{|c|}{ Observer: Walter Kovacs } \\
\hline ID & Length & Yield \\
\hline 38681 & 80 & DNA: 100 \\
\hline & & RNA: ?? \\
\hline 10034 & $\mathbf{0 . 1}$ & DNA: 122 \\
\hline & & RNA: none \\
\hline 80260 & 19 & DNA: 88 \\
\hline & & RNA: 72 \\
\hline
\end{tabular}

NOTE: italics $=\mathrm{cm}$, bold $=\mathrm{m}$

\begin{tabular}{|r|r|l|}
\hline \multicolumn{3}{|c|}{ Date: Oct 1, 1992} \\
\hline \multicolumn{3}{|c|}{ Observer: Reggie Long } \\
\hline ID & Length & Yield \\
\hline 10545 & 1m & DNA: 50 \\
\hline & & RNA: 10 \\
\hline 75262 & $88 \mathrm{~cm}$ & DNA: 61 \\
\hline & & RNA: 40 \\
\hline 21221 & $0.9 \mathrm{~m}$ & DNA: 44 \\
\hline & & RNA: 36 \\
\hline
\end{tabular}

(B)

\begin{tabular}{|r|r|r|r|r|r|r|}
\hline ID & Date & Observer & Length & Len_metric & Nucleotide & Yield \\
\hline 38681 & $1984-07-01$ & Walter Kovacs & 80 & $\mathrm{~cm}$ & DNA & 100 \\
\hline 38681 & $1984-07-01$ & Walter Kovacs & 80 & $\mathrm{~cm}$ & RNA & NA \\
\hline 10034 & $1984-07-01$ & Walter Kovacs & 0.1 & $\mathrm{~m}$ & DNA & 122 \\
\hline 10034 & $1984-07-01$ & Walter Kovacs & 0.1 & $\mathrm{~m}$ & RNA & 0 \\
\hline 80260 & $1984-07-01$ & Walter Kovacs & 19 & $\mathrm{~cm}$ & DNA & 88 \\
\hline 80260 & $1984-07-01$ & Walter Kovacs & 19 & $\mathrm{~cm}$ & RNA & 72 \\
\hline 10545 & $1992-10-01$ & Reggie Long & 1 & $\mathrm{~m}$ & DNA & 50 \\
\hline 10545 & $1992-10-01$ & Reggie Long & 1 & $\mathrm{~m}$ & RNA & 10 \\
\hline 75262 & $1992-10-01$ & Reggie Long & 88 & $\mathrm{~cm}$ & DNA & 61 \\
\hline 75262 & $1992-10-01$ & Reggie Long & 88 & $\mathrm{~cm}$ & RNA & 40 \\
\hline 21221 & $1992-10-01$ & Reggie Long & 0.9 & $\mathrm{~m}$ & DNA & 44 \\
\hline 21221 & $1992-10-01$ & Reggie Long & 0.9 & $\mathrm{~m}$ & RNA & 36 \\
\hline
\end{tabular}

Figure 2. Example of (A) common errors in data management and (B) corresponding rearrangement of the same data to simplify reproducible data wrangling and analysis. Note that colours are added to show link between data in A and B and do not appear in the final text-based file (e.g. TXT, CSV, TSV).

In addition to eliminating empty spaces and conforming to the 1 cell = 1 characteristic rule, each column should be homogenous; that is, a column should contain only a single type of data. Data types are typically integers (whole numbers between -4324 and 10000002), floating point numbers (non-integer numbers), boolean values (just two states: True or False; 1 or 0 ) or strings (alphanumeric characters). As shown in Figure 2, each column should be homogeneous - that is, all entries contain the same type of data. For example, if length measurements were made in $\mathrm{cm}$ and $m$, then the measurements ' $1 \mathrm{~m}$ ' and ' $10 \mathrm{~cm}$ ' should be separated into integer and string columns to avoid heterogenous data, as shown in Figure 2. Using different values for the same entity also creates undesirable heterogeneity in a data column, for example alternating between ' $F$ ', ' $f$ ', 'Female' and 'female', which are interpreted as different values by analysis software. Notes about individual observations (i.e. rows) or the overall dataset (i.e. metadata) should not be hard-coded as stand-alone rows in datasheets, as shown in Figure $2 \mathrm{a}$, but instead encoded as columns or included in a separate file called readme.txt, notes.txt, metadata.txt, or something analogous. A metadata file (e.g. metadata.txt) should include a list of each column and all variable names, with brief descriptions and units of measurement. Similarly, associated files such as images or DNA sequences (e.g. FASTA or FASTQ) can be indexed with reference columns containing file names or ID codes that match to corresponding image file names or DNA sequence IDs. Finally, data should be saved in a non-proprietary, human readable text file format to ensure longevity (e.g. TXT, TAB or CSV file).

Table 1. Summary of recommended data standards

$$
\text { Standard }
$$

Example 
Consistent naming

Appropriate data types

Homogenous data types

Human readable, nonproprietary formats

Appropriate null coding (no empty cells)

Appropriate variable names
Use a single value for representing a female individual, rather than inconsistent use of, for example, ' $F$ ', ' $f$ ', 'Female' and 'female'. Integer (42), Float (3.14), String ('cat'), Boolean (True/False), or Date (YYYY-MM-DD) where appropriate.

Don't mix data types in a single column. For example, if you measured in $\mathrm{cm}$ and $\mathrm{mm}$, use one integer column for the measurement and a separate string column for the unit of measurement. Data file can be opened using a standard text editor. For clarity, these files are typically saved with the suffix .txt, .csv (comma-separated) or .tsv (tab-separated). Use 0 for observed zeros but $\mathrm{NA}^{1}, \mathrm{NaN}$, or NULL for missing data.

Short but informative variable names, perhaps combining multiple words (e.g. flowering time) using short-forms with a period (flwr.t), underscore (flwr_t) or capital letter (flwrT).

${ }^{1} \mathrm{NA}$ is the preferred value for missing data in $\mathrm{R}$.

In addition to the basic standards outlined above, multiple schema are available to standardize and share data. For example, Ecological Metadata Language (Fegraus et al. 2005) is a data schema which puts no restraints on the kinds of data collected but requires comprehensive documentation of data through metadata standards. Darwin Core (Wieczorek et al., 2012) is another schema that uses each row as a record and has a variety of standardized column names. The resulting data package can then be uploaded and shared on online repositories such as DataONE, Dryad, and the Knowledge Network for Biocomplexity (Andelman et al., 2004; Greenberg, 2009; Michener et al., 2012). Biodiversity repositories such as the Global Biodiversity Information Facility accept Darwin Core datasets with metadata files. 
baRcodeR with PyTrackDat

\section{PyTrackDat Pipeline}

The PyTrackDat pipeline (https://github.com/ColauttiLab/PyTrackDat) contains two main Python scripts: The first script analyzes text-based data files, such as the data.frame object generated from baRcodeR, to generate a design file as described below. The second script uses a design file to generate a web application for sample tracking and data collection. Although designed to integrate with baRcodeR, both scripts in PyTrackDat can be run independently on user-defined input files.

The first part of the PyTrackDat pipeline is an optional DataAnalyzer script that assists with implementation of the data standards outline in the previous section. It can be run with a single CSVformatted input file, or a series of CSV files linked by one or more column names (e.g. sampleID). In the latter case, the code will remap fields (i.e. columns) from multiple data tables into a single, more detailed database format with associated metadata. This format specifies field properties such as data type, acceptable values, and whether fields can contain NULL values (Fig. 3). The design file should be inspected manually using a text editor to look for errors (e.g. inconsistent data types) and to add descriptions about the data. The edited design file serves two main purposes: (i) archiving data types and other metadata that isn't easily stored in a spreadsheet format; (ii) rigidly defining fields and links among data tables to create a relational database.

The second script is WebBuilder, which generates a web-app for data collection based on input from a design file. The design file can be a completed version of the file generated from the DataAnalyzer script or a de-novo design file written by the user using a text editor. Based on this input, the second script in the PyTrackDat pipeline creates a zipped folder containing the packaged web application, ready to be deployed (see README.md at https://github.com/ColauttiLab/PyTrackDat).

\begin{tabular}{|c|c|c|c|c|c|c|c|c|c|c|c|c|}
\hline 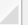 & A & в & c & D & E & $\mathrm{F}$ & G & $\mathrm{H}$ & 1 & 」 & $\mathrm{k}$ & $\mathrm{L}$ \\
\hline 1[ & samples & Inew field name & data type & nullable? & null values & default & description & \multicolumn{2}{|c|}{ additional fields... } & & & \\
\hline 2 & Date & date & integer & FALSE & & & Ifill me in! & & & & & \\
\hline 3 & Date2 & date2 & date & FALSE & & & !fill me in! & & & & & \\
\hline 4 & Year & year & integer & FALSE & & & !fill me in! & & & & & \\
\hline 5 & Season & season & text & FALSE & & & !fill me in! & \multicolumn{3}{|c|}{12 fall; spring; winter } & & \\
\hline 6 & Site ID & site_id & integer & TRUE & & & !fill me in! & & & & & \\
\hline 7 & Gear Type & gear_type & text & TRUE & & & Ifill me in! & \multicolumn{4}{|c|}{$14 \mathrm{CM}$; Jig; MM; SS; SS/CM ?; u } & \\
\hline 8 & Sample\# & sample & unknown & FALSE & & & Ifill me in! & & & & & \\
\hline 9 & Spp. Code & spp_code & text & FALSE & & & Ifill me in! & \multicolumn{5}{|c|}{18 arch; arch/lktr; cod; cspp; fldr; lktr; lkwh; sscu } \\
\hline 10 & Species ID by Geraint & species_id_by_geraint & text & FALSE & & & Ifill me in! & & & & & \\
\hline $11:$ & Species ID Confidence by Geraint & species_id_confidence_by_geraint & unknown & FALSE & & & !fill me in! & & & & & \\
\hline 12 & AGE & age & integer & TRUE & & & !fill me in! & & & & & \\
\hline 13 & Yearclass & yearclass & integer & TRUE & & & Ifill me in! & & & & & \\
\hline 14 & Mesh size (mm) & mesh_size_mm & unknown & FALSE & & & Ifill me in! & & & & & \\
\hline 15 & Caught Dead or Alive & caught_dead_or_alive & text & TRUE & & & !fill me in! & & $a ; d ; u$ & & & \\
\hline 16 & Photo & photo & boolean & TRUE & u & & !fill me in! & & & & & \\
\hline 17 & Length Type & length_type & text & FALSE & & & !fill me in! & & $\mathrm{fl} ; \mathrm{n} ; \mathrm{tl}$ & & & \\
\hline 18 & Length $(\mathrm{mm})$ & length_mm & integer & TRUE & & & !fill $m e$ in! & & & & & \\
\hline 19 & Round weight (g) & round_weight_g & unknown & FALSE & & & Ifill me in! & & & & & \\
\hline 20 & Sex & sex -20 & text & TRUE & & & Ifill me in! & & $f ; m ; n ; u$ & & & \\
\hline 21 & Gonad wt.(g) & gonad_wtg & unknown & FALSE & & & Ifill $\mathrm{me}$ in! & & & & & \\
\hline 22 & Repr. Stat & repr_stat & text & TRUE & & & Ifill $\mathrm{me}$ in! & & $i ; m ; n ; r$ & & & \\
\hline 23 & Oto. & oto & unknown & FALSE & & & !fill me in! & & & & & \\
\hline 24 & Sc. & sc & boolean & FALSE & & & Ifill me in! & & & & & \\
\hline 25 & Fin & fin & boolean & FALSE & & & !fill me in! & & & & & \\
\hline 26 & Fish kept separate to prevent slime cross contam. & fish_kept_separate_to_prevent_slime_cross_contam & text & TRUE & & & Ifill me in! & & n; new $\mathrm{m}$ & & & \\
\hline 27 & Comments & comments & text & FALSE & & & Ifill me in! & & & & & \\
\hline 28 & Inuktitut ID & inuktitut_id & text & FALSE & & & !fill me in! & & & & & \\
\hline 29 & Sample Type & sample_type & text & TRUE & & & !fill me in! & & & & & \\
\hline
\end{tabular}

Figure 3. A sample design file generated from the DataAnalyzer script in the PyTrackDat pipeline. Data types are automatically inferred for each data column in the CSV file, with 'unknown' indicating fields that should be investigated by the user. The number of different options for text variables are shown under 'additional fields', along with some examples.

\section{Implementation}

To limit errors while improving repeatability of methods and facilitating reproducibility of downstream analysis, we suggest two alternative strategies for data collection and curation. For small projects with one or a few investigators working together, such as a student thesis project or for a focused technical 
baRcodeR with PyTrackDat

report, a basic data management framework proposed above may be appropriate. Further considerations are needed for large, complex databases used by multiple investigators from different research groups. Although it is theoretically possible to encode everything in a single spreadsheet format, this may require frequent edits by multiple users, resulting in an unstable dataset. Instead, data can be organized into separate files linked by identifying columns (e.g. sample, collector, location, date). These are referred to as 'relational databases' and many software packages are available to handle such data, including dBase (dBase LLC, Binghampton; http://www.dbase.com), FileMaker Pro (FileMaker, Santa Clara; http://www.filemaker.com), Google Fusion Tables (Alphabet, Santa Clara, http://drive.google.com), LibreOffice Base (The Document Foundation, Berlin, http://www.libreoffice.org), and MySQL (Oracle, Redwood City, http://www.mysql.com).

To facilitate creation and collection of both 'basic' and 'relational' data, baRcodeR and PyTrackDat were designed to facilitate sample management and data collection according to the standards outlined in Section 2, above. These standards include generation of code and text files to document sample ID, data management decisions, and data characteristics as an important but underappreciated step in open, repeatable, and reproducible science. The utility of our design philosophy can best be illustrated by three case studies, outlined briefly below, which involve collaborative projects spanning large geographical regions and involving dozens to hundreds of collaborators studying microbes, plants and animals using a large variety of conventional and cutting-edge high-throughput technologies. These projects are illustrative as they represent three different stages of development: Example 1 predates baRcode $R$ and inspired many of its features, example 2 was co-developed baRcodeR and PyTrackDat, and example 3 is a new project informed by these standards and inspiring further refinement.

\section{Example 1: GGMFS}

The 'Global garlic mustard field survey' (GGMFS) is a coordinated, distributed experiment in evolutionary ecology involving >150 collaborators from 16 countries spanning Europe and North America (www.garlicmustard.org). The overall goal of the project is to focus research effort on a single focal species, Alliaria petiolata (garlic mustard), to better understand how natural and anthropogenic processes affect genotype, environment and genotype-by-environment effects on phenotypic traits and ecological interactions at local $(<10 \mathrm{~m})$ to global spatial scales. Details of the project, including protocols and design philosophy are discussed elsewhere (Colautti et al., 2014). Here we elaborate on the logistical challenges that arose from this large collaborative project and how it informed the design philosophy applied to baRcodeR and PyTrackDat.

The project began with a relatively simple field sampling protocol to collect seeds and to measure plant size and fecundity, population density, herbivory and pathogen damage, as well as characteristics of the surrounding environment (protocol https://doi.org/10.6084/m9.figshare.729274). To link seed collections with their associated data, we implemented a sample naming standard using the general format:

\section{JDNYC1-3-20}

Where 2010 is the year of collection, JD are the initials of the collector, NYC is a 3-letter location code provided by the collector, 1 is the first population sampled at that location, and 3-20 denotes a sample from $3 \mathrm{~m}$ and $20 \mathrm{~cm}$ along a $10 \mathrm{~m}$ transect. Participants also took canopy photos at 3 points along the transect, and the resulting image files were saved with file names incorporating the population code and transect location. Although a standard protocol was implemented, minor differences among the $>150$ 
baRcodeR with PyTrackDat

different academic participants posed challenges to collect and curate standardized data. To facilitate data entry, we used an online survey service (surveymonkey.org). A web-based portal simplified data aggregation but in several cases the participants deviated from established protocol in their choice of measurement format. This led to a variety of ambiguous entries that took three years to correct. Perhaps the most problematic issue was the entry of GPS coordinates, which were entered in every format available: DMS: 41²4'12.2"N 2¹0'26.5"E, DMM: 41 24.2028, 2 10.4418, DD: 41.40338, 2.17403, and several confusing hybrids (e.g. 41.24.2028). To correct these issues, we wrote custom code in $R$ using regular expressions and sent hundreds of emails to clarify contributed data. Enforcing homogeneous data standards from the beginning would have saved hundreds of hours of work.

Current studies associated with the project use the archived seed collections for laboratory and field experiments measuring a variety of phenotypic traits, genotyping using high-throughput sequencing methods, and soil microbial feedbacks. The use of a standard ID code allows a quick link back to any of the original field measurements. Once published, data from current and future studies can be linked via the biologically-informed ID format and quickly accessed for further analysis using the relational database framework described above.

\section{Example 2: TSFN}

From the inception of the Genome Canada project, "Towards a sustainable fishery for Nunavummiut" (TSFN) it was understood that this collaborative effort between more than half a dozen research labs would be necessary to successfully track thousands of samples collected over an area of $2500 \mathrm{~km}^{2}$ under the challenging conditions of the high Arctic in all seasons. Selected data must be accurate, repeatable and reliable for upload to a digital geographic Atlas, providing a rich and accessible resource for other researchers and Inuit community members. We posited that digital barcoding would provide the only reliable means to track each and every fish sample (fin clips, right and left otoliths, organ biopsies, gonads, skin mucous, intestinal sections, parasites, scales, muscle for contaminant assays and genetics), as well as the metadata associated with each sample (e.g. geographic location, date, name of fisher, net size, net set hours, species, sex, weight, length, photograph). Efforts to purchase software were abandoned as we could not find an affordable solution that was flexible enough to quickly generate biologically meaningful sample ID tags and to accommodate a diverse range of label sizes for various sub-sampling containers.

Using baRcodeR, we created fish-specific ID codes with 2D barcodes printed on waterproof paper and vinyl labels in convenient sizes such as $10 \times 5 \mathrm{~cm}$ and $4.5 \times 1.3 \mathrm{~cm}$; Uline, Milton, ON). These were used to label cryotubes, centrifuge tubes, and a range of sizes of re-sealable plastic bags and manila envelopes (Fig. 4). These sample containers were aggregated into a large plastic bag $(27 \times 27 \mathrm{~cm})$, affixed with the fish-specific barcode mounted on a white sheet bearing a $9.5 \mathrm{~cm}$-scale line beneath the lower barcodes. The container with scale bar was included in photographs of the fish as a redundant check on the accuracy of the reported length measurements. Barcodes were initially hand-scanned (reader CR1421-PKU, Code Corp.) directly into a spreadsheet in Microsoft Excel (ver. 16.16.1) with descriptors added manually. However, data heterogeneity resulting from project participants who were not familiar with the recommended data guidelines (Table 1) prompted development of PyTrackDat. For example, although the length of each fish is routinely measured from the head to the center of the tail fork (fork length), some lengths were reported as head to the end of the squeezed tail (total length); this necessitated the addition of a separate column for total or fork length designations to avoid heterogenous data (see Section 3 guidelines on data standards). The use of the PyTrackDat pipeline to 
baRcodeR with PyTrackDat

create an online data collection and tracking system facilitated data entry according to above standards by multiple users. Data are currently curated by a small number of experienced users before sharing with other collaborators.

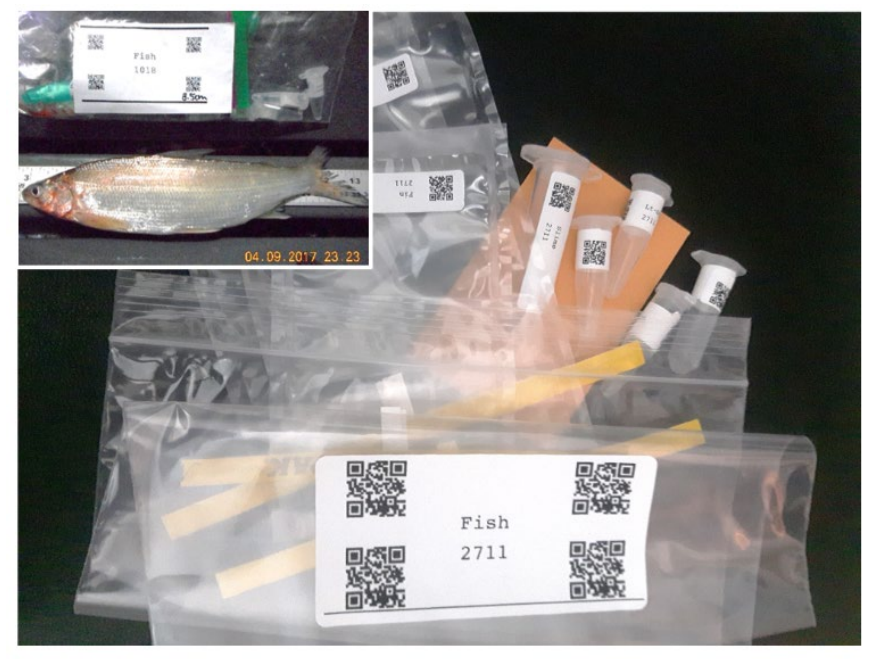

Figure 4. Sampling kit demonstrating the use of baRcodeR to improve data integrity and repeatability of sampling methods. A sample bag for each fish is shipped to the field; each bag contains labelled containers for various tissue subsamples. In this example, separate labels were created with barRcodeR to encode the same fish ID \#2711 into the bag containing the fish, as well as all subsamples associated with it. Inset shows a cropped archival picture of fish \#1018, which visually links a fish ID code to its collection date and time, fish length and species ID.

\section{Example 3: BEARWATCH}

The BEARWATCH project seeks to develop a community-driven program to facilitate ongoing monitoring of polar bears across the Canadian Arctic, combining Inuit traditional knowledge with a toolkit for characterizing key aspects of population health. In addition to extensive consultation with northern communities and collation of existing traditional knowledge, Phase 1 of the project involves creating a baseline map of genetic structure by genotyping thousands of individuals harvested by indigenous hunters or biopsied by territorial governments over several decades. Subsamples from archived tissue arrived either fixed with 95\% ethanol in Eppendorf vials, dried in an envelope (skeletal muscle), or as a preserved biopsy plug (with associated fat, skin, hair, and muscle). All have either a NT (Northwest Territories) or NU (Nunavut) field sample number previously assigned by territorial governments (e.g. NT_100177, NT_100178, NU_L26303, NU_L35160). Associated metadata include geographical coordinates (decimal degree latitude and longitude), management unit (one of 19 worldwide indicated by a 2-letter code; e.g. Gulf of Boothia=GB, Lancaster Sound=LS; see Obbard et al., 2010), collection date (Year-Month-Day), sex (M, F), and age (Adult, Subadult, 2Yearling). Upon receiving samples to our Biosafety Level 2 laboratory, each is assigned a barcode generated using uniqID_maker() (Um-000001, Um-000002, Um-000003). The prefix ' $U m$ ' is for Ursus maritimus, followed by 6 digits anticipating a continuous monitoring system that can accommodate continued influx of samples for the foreseeable future.

We are using a reduced-representation genotype-by-sequencing approach (double-digest RAD-Seq; Peterson et al., 2012) to generate large panels of Single Nucleotide Polymorphism (SNP) data that will be 
baRcodeR with PyTrackDat

used for quantifying population structure and forensic genotyping. From Illumina sequencing runs, each individual bear will have an associated FASTQ file containing millions of individual sequence short reads, with details on instrument, run date, read quality, etc. As these data are too extensive to store in our master database, unique FASTQ file names (barcode_FASTQ.txt) will be listed as a string within one variable field for each bear referencing the pertinent FASTQ files stored elsewhere on our servers.

Phase 2 of the project will analyze contaminant loads, stable isotope profiles, and diet using DNA barcoding of polar bear scat collected across the Canadian Arctic (an area larger than Western Europe) by Inuit hunters and other agencies. Polar bear DNA from each scat sample will also be analyzed using a genetic toolkit we are developing to determine the sex and genetic identity from a subset of 182 biallelic SNPs identified in Phase 1. Each scat sample will again have associated metadata that includes geographical coordinates, management unit, and collection date. Each scat will be subsampled four times for separate genomic, contaminant, stable isotope, and contaminants analyses to be done in laboratories at three different institutions. We will use uniqID_hier_maker() to generate unique identifiers for tracking individual subsamples based on a pre-defined numbering system (1-genomics, 2contaminants, 3-stable isotopes, 4-diet) - e.g. Um-000113-1, Um-000113-2, Um-000113-3, Um-0001134).

As this project ultimately envisions continued community-based scat sampling over decades, we anticipate receiving multiple scat samples belonging to the same individuals obtained in distinct seasons, years, and locations - the range of individual polar bears can encompass areas $>100,000 \mathrm{~km}^{2}$. (Ferguson et al., 1999). This will allow us to assess movement patterns and changes in contaminant load, diet, and trophic level for individual bears over time and space. However, we will not know initially whether we have sampled the same individual or close relatives until we have created the SNP profile, and either tested for genotype matches (e.g., Jin et al., 2017) or estimated relatedness using a program like SNPRelate Version 1.14.0 (Zheng et al., 2012). A separate data table will contain a pairwiserelatedness matrix of all scat samples, which will allow for later grouping of barcodes linking individuals based on a threshold similarity in the relatedness matrix, as well as samples representing likely relatives (e.g. full-sibs, half-sibs, parent-offspring). Overall, this project demonstrates the utility of baRcodeR with PyTrackDat when designing sampling strategies that must link heterogeneous data across researchers and collection dates, with the flexibility to add new data fields based on analyses that occur long after samples have been collected and processed.

\section{Conclusion}

The rigour of scientific theories in the biological sciences rest on fundamental concepts of repeatable experimental methods coupled with reproducible data analysis. Many key and fundamental results of published research in the sciences and social sciences are not reproducible (Baker, 2016), due at least partly to insufficient published detail about experimental methods, data collection and statistical analysis (loannidis, 2005). The use of open-source tools for documenting data wrangling and analysis in scientific studies are important steps towards improving reproducibility and transparency of data published research. To complement these tools, we have introduced baRcodeR and PyTrackDat to improve transparency and repeatability upstream of data wrangling and analysis. This includes documentation of sampling design, data collection, and dataset curation. Using examples, we have demonstrated how these tools are particularly useful to large-scale collaborative research projects in 
baRcodeR with PyTrackDat

biology, studies using high-throughput methods for data collection, and more generally for researchers striving to improve the documentation, repeatability and reproducibility of their scientific endeavours.

\section{Acknowledgements}

We thank E. Bao for contributions to baRcodeR code, Dr. P. V.C. de Groot for his efforts in the TSFN fish and BEARWATCH sampling as well as E. Jensen, R. Clemente-Carvalho, K. Flock, the Gjoa Haven, NU community residents, the Hunters and Trappers Association (HTA), the Government of Nunavut, Natural Sciences and Engineering Research Council (Canada) and large-scale Genome-Canada Projects, and these projects' associated supporters. We would like to thank all sampling personnel that provided invaluable help in the field, along with our Inuit guides who were integral to the Arctic sampling efforts. We also thank. We further acknowledge that Queen's University is situated on traditional Anishinaabe and Haudenosaunee territory and we are grateful to be able to be live and learn on these lands.

\section{Literature Cited}

Andelman, S.J., Bowles, C.M., Willig, M.R., and Waide, R.B. (2004). Understanding environmental complexity through a distributed knowledge network. BioScience 54, 240-246.

Baker, M. (2016). 1,500 scientists lift the lid on reproducibility. Nat. News 533, 452.

Blagoderov, V., Kitching, I.J., Livermore, L., Simonsen, T.J., and Smith, V.S. (2012). No specimen left behind: industrial scale digitization of natural history collections. ZooKeys 133-146.

Borer, E.T., Seabloom, E.W., Jones, M.B., and Schildhauer, M. (2009). Some simple guidelines for effective data management. Bull. Ecol. Soc. Am. 90, 205-214.

British Ecological Society (2014). A Guide to Data Management in Ecology and Evolution.

Chang, W., Cheng, J., Allaire, J., Xie, Y., and McPherson, J. (2018). Shiny: Web Application Framework for R.

Colautti, R., Franks, S.J., Hufbauer, R.A., Kotanen, P.M., Torchin, M., Byers, J.E., Pyšek, P., and Bossdorf, O. (2014). The Global Garlic Mustard Field Survey (GGMFS): challenges and opportunities of a unique, large-scale collaboration for invasion biology. NeoBiota 21, 29-47.

Copp, A.J., Kennedy, T.A., and Muehlbauer, J.D. (2014). Barcodes are a useful tool for labeling and tracking ecological samples. Bull. Ecol. Soc. Am. 95, 293-300.

European Commission European Research Council (ERC) Frontier Research Grants Information for Applicants to the Advanced Grant 2017 Call.

Ferguson, S.H., Taylor, M.K., Born, E.W., Rosing-Asvid, A., and Messier, F. (1999). Determinants of home range size for polar bears (Ursus maritimus). Ecol. Lett. 2, 311-318.

Government of Canada, I. DRAFT Tri-Agency Research Data Management Policy For Consultation Science.gc.ca. 
Greenberg, J. (2009). Theoretical considerations of lifecycle modeling: An analysis of the Dryad Repository demonstrating automatic metadata propagation, inheritance, and value system adoption. Cat. Classif. Q. 47, 380-402.

Ioannidis, J.P.A. (2005). Why most published research findings are false. PLOS Med. 2, e124.

Jin, Y., Schäffer, A.A., Sherry, S.T., and Feolo, M. (2017). Quickly identifying identical and closely related subjects in large databases using genotype data. PLOS ONE 12, e0179106.

Kluyver, T., Ragan-Kelley, B., Pérez, F., Granger, B., Bussonnier, M., Frederic, J., Kelley, K., Hamrick, J., Grout, J., Corlay, S., et al. (2016). Jupyter Notebooks - a publishing format for reproducible computational workflows. Stand Alone 87-90.

List, M., Schmidt, S., Trojnar, J., Thomas, J., Thomassen, M., Kruse, T.A., Tan, Q., Baumbach, J., and Mollenhauer, J. (2014). Efficient sample tracking with OpenLabFramework. Sci. Rep. 4, 4278.

Michener, W.K., Allard, S., Budden, A., Cook, R.B., Douglass, K., Frame, M., Kelling, S., Koskela, R., Tenopir, C., and Vieglais, D.A. (2012). Participatory design of DataONE-Enabling cyberinfrastructure for the biological and environmental sciences. Ecol. Inform. 11, 5-15.

National Science Foundation Open Government Plan 3.5.

Obbard, M.E., Thiemann, G.W., Peacock, E., and DeBruyn, T.D. (2010). Polar Bears: Proceedings of the 15th Working Meeting of the IUCN/SSC Polar Bear Specialist Group, 29 June-3 July 2009, Copenhagen, Denmark (IUCN).

Oliphant, T.E. (2007). Python for scientific computing. Comput. Sci. Eng. 9, 10-20.

Peterson, B.K., Weber, J.N., Kay, E.H., Fisher, H.S., and Hoekstra, H.E. (2012). Double digest RADseq: An inexpensive method for de novo SNP discovery and genotyping in model and non-model species. PLOS ONE 7, e37135.

R Core Team (2018). R: A Language and Environment for Statistical Computing (Vienna, Austria: R Foundation for Statistical Computing).

RStudio Team (2016). RStudio: Integrated Development Environment for R (Boston, MA: RStudio, Inc.).

Wieczorek, J., Bloom, D., Guralnick, R., Blum, S., Döring, M., Giovanni, R., Robertson, T., and Vieglais, D. (2012). Darwin Core: An evolving community-developed biodiversity data standard. PLOS ONE 7, e29715.

Wu, Y., and Colautti, R.I. (2018a). baRcodeR: Labelling, Tracking, and Collecting Data from Biological Samples. Comprehensive R Archive Network (CRAN). https://cran.r-project.org/package=baRcodeR

Wu, Y., and Colautti, R.I. (2018b). baRcodeR Vignette. Comprehensive R Archive Network (CRAN). https://cran.r-project.org/package=baRcodeR/baRcodeR.pdf

Wu, Y., and Colautti, R.I. (2018c). baRcodeR Cheat-Sheet. FigShare. https://doi.org/10.6084/m9.figshare.7043309.v4 
bioRxiv preprint doi: https://doi.org/10.1101/457051; this version posted October 30, 2018. The copyright holder for this preprint (which was not certified by peer review) is the author/funder, who has granted bioRxiv a license to display the preprint in perpetuity. It is made available under aCC-BY-NC-ND 4.0 International license.

baRcodeR with PyTrackDat

Zheng, X., Levine, D., Shen, J., Gogarten, S.M., Laurie, C., and Weir, B.S. (2012). A high-performance computing toolset for relatedness and principal component analysis of SNP data. Bioinformatics 28 , 3326-3328. 